\title{
Base Station Coordination towards an Effective Inter-cell Interference Mitigation
}

\author{
Moubagou Deflandre Bertrand and Chang Yong Yu \\ Beijing University of Posts and Telecommunication, Haidian10 Xitucheng, \\ 100876, China \\ deflandb@yahoo.fr,yychang@bupt.edu.cn
}

\begin{abstract}
Improving cell-edge multi-user performance in $3 G P P$ Long Term EvolutionAdvanced networks is becoming a serious concern for the next generation wireless networks. This paper proposes Base Station Coordination as a promising solution to tackle Inter-Cell Interference especially for users located at the cell border. The celledge spectral efficiency is actually a more important performance index in practice due to the high-speed transmission expected in all the coverage area of a modern cellular network. Considering the preciousness of resources, our paper provides a better idea to work towards developing a new downlink and uplink transmission scheme, which could allow multi-user transmissions on the same resource to increase the utilization efficiency. We apply the pre-coding on the coordinated evolved Node B to achieve simultaneous multi-user transmissions on the same frequency band. The pre-coding matrix designed is investigated and results show that Zero-forcing precoding of Base Station Coordination reaches significant capacity enhancement compared to the conventional network in case of a relatively high Signal-to-NoiseRatio.
\end{abstract}

Keywords: Inter-cell interference, Base station coordination, Zero-forcing precoding, LTE-Advanced, SNR

\section{Introduction}

Cellular networks have been experiencing a fast development in recent years. After a great success of GSM and the communalization of $3 \mathrm{G}$ networks, International Telecommunication Union (ITU) proposed a new global standard framework, International Mobile Telecommunication (IMT)-advance to build future generation cellular networks which can fulfill the ever growing demands on capacities, motilities and so on. $3^{\text {rd }}$ Generation Partnership Project (3GPP) Long Term Evolution (LTE-A) [1] is one of candidates and even surpasses some requirements of IMT-Advanced. These requirements aim to satisfy not only the peak data rate and spectral efficiency but also the cell-edge spectral efficiency.

The cell-edge spectral efficiency is actually a more important performance index in practice due to ubiquitous high-speed transmissions expected in all the covered area of modern cellular network.

The long lasting problem on wireless system design is how to achieve a higher system capacity with lower resource consumption. Here the term resource refers to time, frequency and power, which can be allocated by the network operators.

Specifically, in the cellular networks, we always face a challenging problem that the performance of cell-edge users is much worse than the users in the cell center. The first reason of the performance degradation of the cell-edge users can be related to the long distance between the Base Station (eNB) and the User Equipment (UE) and the limited power of transmit antennas. It is known that the signal strength decreases proportionally 
to the exponentiation of the distance in the wireless communications. With the limited power of transmit antennas, the receiver would get very weak useful signal in a noisy environment, and the low receive SNR results in a low data rate [2].

The second reason is the inter-cell interference. In both uplink and downlink phases, transmissions in one cell would interfere with the transmissions in the other neighbor cells if they are operated on the same resource. According to the problem statement described above, if we want to improve the cell edge performance, we should find out an efficient solution to tackle two problems: how to enhance the wireless links and how to cancel the inter-cell interference.

Traditionally, the scheduler would assign orthogonal resource to the adjacent cells to avoid the inter-cell interference [3-4]. However, based on the multi-user information theory, particularly the multi-user MIMO technology, base station coordination can achieve multi-user transmission on each allocated resource block, which would significantly enlarge the system capacity [5].

This paper proposes a better idea to work towards developing a new transmission scheme, which could allow multi-user transmissions on the same resource to increase the utilization efficiency. We use the term base station coordination to denote this kind of joint multi-cell multi-user transmission scheme. Its purpose is to evaluate the base station coordination technology. With multi-cell processing, the adjacent eNBs can form a virtual antenna array and then the system can then be model as MIMO MAC and MIMO BC in uplink and downlink phases respectively [6]. With the tools provided by the multi-user information theory, we can calculate the theoretical bound of multi-user MIMO sum-rate capacity. Zero-forcing pre-coding is chosen to realize multi-user transmissions in the downlink phase in the base station coordination scheme discussed in our report. Note that in both uplink and downlink phases, the cells can operate simultaneously on the same frequency because the inter-cell interference is cancelled by the Zero Forcing (ZF) precoding procedure on the eNBs.

The paper is organized as follows: In section II, the analysis both in downlink and uplink transmission and their outage probabilities are formulated. In section III, the numerical results compared to the conventional network are shown. Finally, conclusions are drawn in section IV.

\section{System Model}

\subsection{Conventional Network}

In a conventional cellular, each eNB serves UEs in its own cell, with neither relays nor any kinds of coordination. In an interference-free network, the resources allocated for the different cells should be orthogonal. Assuming that transmissions in adjacent cells are time divided, without loss of generality, transmissions are following numerical orders. We establish a conventional network as reference system for the further comparisons. This paper limits the analysis in a simple two-cell network to highlight the performance improvement of the base station coordination and the results can be extended to cases with more cells. In fig. 1red line describes the transmission in the first time slot (for cell 1) and the blue line describes the transmission in the second time slot (for cell 2). 


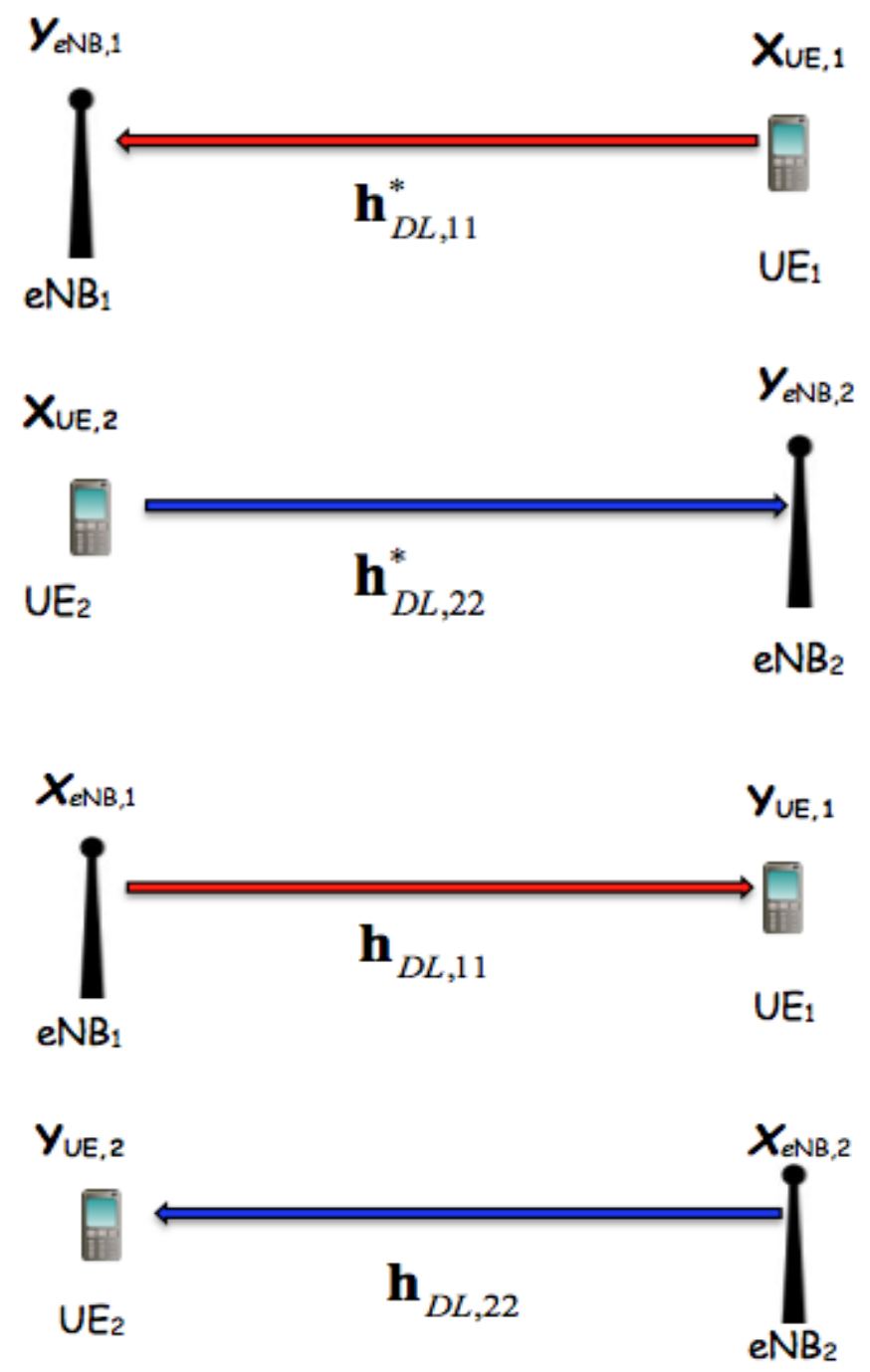

\section{Figure 1. Uplink (above) and Downlink (below) Transmission in a Conventional Network}

For the uplink transmissions, in the first time slot [t], the received signal at $\mathrm{eNB}_{1}$ is

$$
\begin{gathered}
\mathrm{y}_{\mathrm{eNB}_{1}}[\mathrm{t}]=\mathrm{h}_{\mathrm{DL}, 11}[\mathrm{t}] \mathrm{x}_{\mathrm{UE}, 1}[\mathrm{t}]+\mathrm{n}_{\mathrm{eNB}, 1}[\mathrm{t}](1) \\
\mathrm{y}_{\mathrm{eNB}_{2}}[\mathrm{t}+1]=\mathrm{h}_{\mathrm{DL}, 22}[\mathrm{t}+1] \mathrm{x}_{\mathrm{UE}, 2}[\mathrm{t}+1]+\mathrm{n}_{\mathrm{eNB}, 2}[\mathrm{t}+1](2)
\end{gathered}
$$

Assuming the channel gain stays unchanged over two time slots $[t]$ and $[t+1]$, for a unit bandwidth, the sum-rate capacity of this conventional network is the average of rates in the two time slots, which can be estimated according to Shannon-Hartley theorem which pointed out that the capacity of a limited bandwidth Gaussian channel is [7]

$$
\mathrm{C}=\mathrm{B} \log _{2}(1+)(3)
$$

From this equation we can get the sum-rate capacity of the conventional network respectively for the uplink and downlink as 


$$
\mathrm{C}_{\mathrm{C}_{\text {onv }}}^{\mathrm{UL}}=\frac{1}{2}\left(\log _{2}\left(1+\frac{\mathrm{P}_{\mathrm{UE}}}{2}\left|\mathrm{~h}_{\mathrm{DL}, 11}\right|^{2}\right)+\log _{2}\left(1+\frac{\mathrm{P}_{\mathrm{UE}}}{2}\left|\mathrm{~h}_{\mathrm{DL}, 22}\right|^{2}\right)\right)(4)
$$

where a factor $\frac{1}{2}$ is for averaging over two time slots, subscript Conv denotes the conventional network, $P_{U E}$ is total transmit power that UE could be assigned. ${ }^{2}$ denotes the noise power.

The expression of sum-rate capacity is dual in the downlink transmissions, expect replacing the $\mathrm{P}_{\mathrm{UE}}$ with $\mathrm{P}_{\mathrm{eNB}}$, which is the total power could be allocated by the eNB, i.e.

$$
\mathrm{C}_{\mathrm{C}_{\text {onv }}}^{\mathrm{DL}}=\frac{1}{2}\left(\log _{2}\left(1+\frac{\mathrm{P}_{\mathrm{eNB}}}{2}\left|\mathrm{~h}_{\mathrm{DL}, 11}\right|^{2}\right)+\log _{2}\left(1+\frac{\mathrm{P}_{\mathrm{eNB}}}{2}\left|\mathrm{~h}_{\mathrm{DL}, 22}\right|^{2}\right)\right)(5)
$$

\subsection{Base Station Coordination in Two-Cell Case}

Base station coordination enables adjacent cells to work on the same resource block. With multi-cell processing, the scheme can be considered as an extension of MIMO BC with a distributed transmit antenna array in different cells and the inter-cell interference can be cancelled preliminarily on the transmitter side. In the following, we refer to the connection between $m$ the NB and kth UE as direct link (DL) with channel gain $h_{D L, m k}$. The channels in the uplink are assumed to be the conjugate transpose of those in the downlink. $\mathrm{X}_{\mathrm{eNB}, \mathrm{m}}$ and $\mathrm{X}_{\mathrm{UE}, \mathrm{k}}$ are the signals transmitted by the $\mathrm{m}$ the NB and the kth UE, respectively. $\mathrm{y}_{\mathrm{eNB}, 1}$ and $\mathrm{y}_{\mathrm{UE}, \mathrm{k}}$ are the receive signals at the $\mathrm{m}$ the $\mathrm{NB}$ and the kth $\mathrm{UE}$, respectively. $\mathrm{n}_{\mathrm{eNB}, \mathrm{k}}$ and $\mathrm{x}_{\mathrm{UE}, \mathrm{k}}$ are the noises observed at all the $\mathrm{m}$ the NB and the kth UE, respectively with the same variance ${ }^{2}$ [8].

Assuming that there are MeNBs and K UEs in the network and all the devices are equipped with single antenna, the channel is expressed in a $M \quad K$ or $M_{R} \quad K_{T}$ if we count the numbers of the receive and transmit antennas matrix $\mathbf{H}$ that

$$
\mathbf{H}=\left[\begin{array}{cccc}
h_{\mathrm{DL}, 11} & \mathrm{~h}_{\mathrm{DL}, 21} & \cdots & \mathrm{h}_{\mathrm{DL}, \mathrm{M} 1} \\
\mathrm{~h}_{\mathrm{DL}, 11} & \mathrm{~h}_{\mathrm{DL}, 21} & \cdots & \mathrm{h}_{\mathrm{DL}, \mathrm{M} 2} \\
\vdots & \vdots & \ddots & \vdots \\
\mathrm{h}_{\mathrm{DL}, 1 \mathrm{~K}} & \mathrm{~h}_{\mathrm{DL}, 2 \mathrm{~K}} & \cdots & \mathrm{h}_{\mathrm{DL}, \mathrm{MK}}
\end{array}\right]
$$

where $\mathbf{h}_{\mathrm{k}}$ is the channel from all the eNBs to the kth UE and equal to the kth row of $\mathbf{H}$, i.e. 


$$
\begin{aligned}
& \mathbf{h}_{\mathrm{k}}=\left(\begin{array}{cccc}
\mathrm{h}_{\mathrm{DL}, 1 \mathrm{~K}} & \mathrm{~h}_{\mathrm{DL}, 2 \mathrm{~K}} & \cdots & \mathrm{h}_{\mathrm{DL}, \mathrm{MK}}
\end{array}\right) \text { and } \\
& \mathbf{x}_{\mathrm{eNB}}=\left[\begin{array}{c}
\mathrm{x}_{\mathrm{eNB}, 1} \\
\mathrm{x}_{\mathrm{eNB}, 2} \\
\vdots \\
\mathrm{x}_{\mathrm{eNB}, \mathrm{M}}
\end{array}\right] \text { is the transmit signal vector from the eNBs and the total power }
\end{aligned}
$$

of the transmit antennas is limited as $\mathrm{P}_{\mathrm{eNB}}$, i.e. $\left\{\mathbf{x}_{\mathrm{eNB}} \mathbf{x}_{\mathrm{eNB}}\right\} \quad \mathrm{P}_{\mathrm{eNB}}$. And the uplink transmission can be modeled as:

$$
\mathrm{y}_{\mathrm{eNB}}=\mathbf{H} \mathbf{x}_{\mathrm{UE}}+\mathbf{n}_{\mathrm{eNB}}
$$

Where $\mathbf{H}$ is the MAC channel from all the UEs to the eNBs, $\mathbf{x}_{\mathrm{UE}}=\left[\begin{array}{c}\mathrm{x}_{\mathrm{UE}, 1} \\ \mathrm{x}_{\mathrm{UE}, 2} \\ \vdots \\ \mathrm{x}_{\mathrm{UE}, \mathrm{K}}\end{array}\right]$

is the signal vector from the UEs and the total power of the transmit antenna is limited as $\mathrm{P}_{\mathrm{UE}}$

i.e. $\left\{\mathbf{x}_{\mathrm{UE}} \mathbf{x}_{\mathrm{UE}}\right\} \quad \mathrm{P}_{\mathrm{UE}}$ and $\mathbf{n}_{\mathrm{eNB}}=\left[\begin{array}{c}\mathrm{n}_{\mathrm{eNB}, 1} \\ \mathrm{n}_{\mathrm{eNB}, 2} \\ \vdots \\ \mathrm{n}_{\mathrm{eNB}, \mathrm{M}}\end{array}\right]$ is the noise vector which consists of noises at each eNB's receive antenna.

Considering the downlink transmission, since the eNBs are coordinated in the network, which indicates the signal transmitted by each eNB may contain information intended to multiple UEs, the transmit signal vector $\mathbf{x}_{\mathrm{eNB}}$ could be constructed as

$$
\mathbf{x}_{\mathrm{eNB}}=\mathbf{W d}(8)
$$

Where $\mathbf{d}=\left[\begin{array}{c}\mathrm{d}_{1} \\ \mathrm{~d}_{2} \\ \vdots \\ \mathrm{d}_{\mathrm{k}}\end{array}\right]$ is the data symbol vector, in which $\mathrm{d}_{\mathrm{k}}$ is specific data extended for the kth UE, with unit power, i.e. $\quad\{\mathbf{d d}\}=\mathbf{I}$, and 


$$
\mathbf{W}=\left[\begin{array}{cccc}
\mathrm{w}_{11} & \mathrm{w}_{21} & \cdots & \mathrm{w}_{\mathrm{K} 1} \\
\mathrm{w}_{12} & \mathrm{w}_{22} & \cdots & \mathrm{w}_{\mathrm{K} 2} \\
\vdots & \vdots & \ddots & \vdots \\
\mathrm{w}_{1 \mathrm{M}} & \mathrm{w}_{2 \mathrm{M}} & \cdots & \mathrm{w}_{\mathrm{KM}}
\end{array}\right]
$$

is the M Kpre-coding weight matrix. These weights assign the data symbols with different amplitudes and phases (or transmit power). For the kth UE, all the other data symbols $d_{i}(i \quad k)$ are considered as interference. When all the interference is eliminated, the kth UE only receives data symbol $\mathrm{d}_{\mathrm{k}}$, then multi-user transmissions are achieved. This is one of the most important features of the base station coordination technology [9].
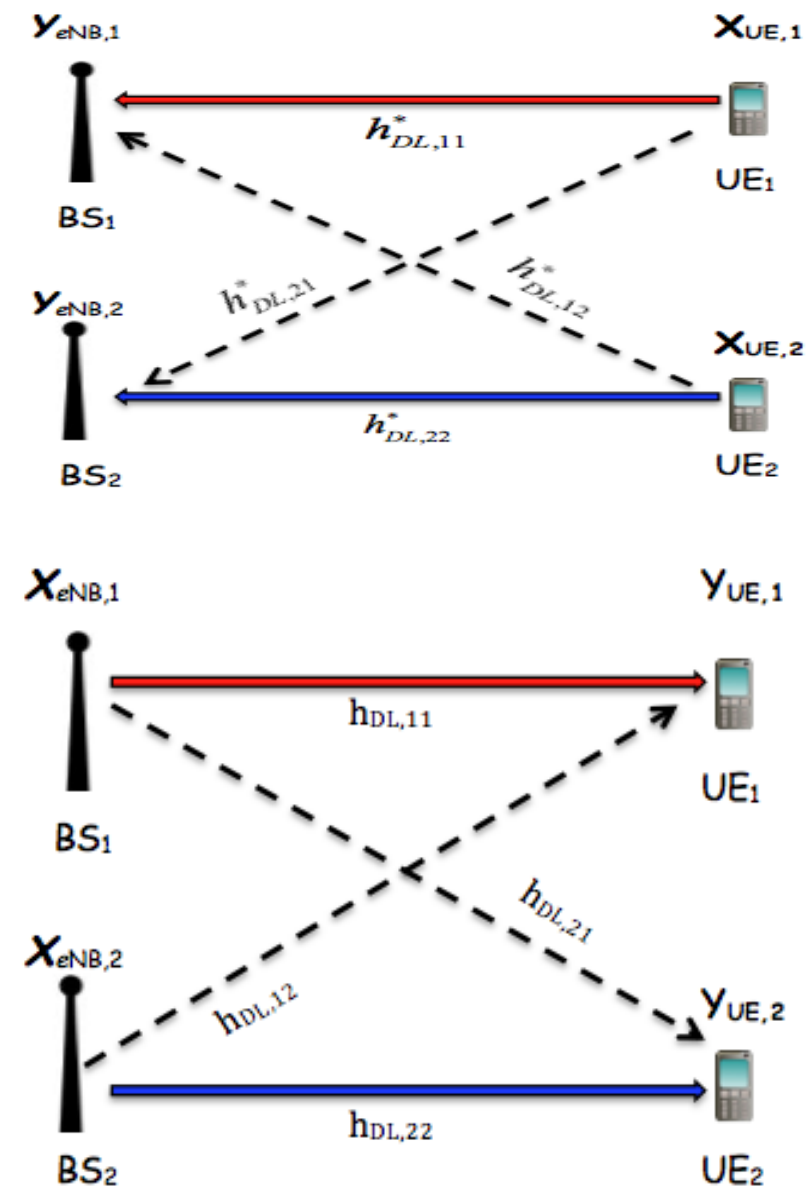

Figure 2. Uplink (above) and Downlink (below) Transmission with Base Station Coordination

Assuming that there are 2 eNBs and 2 UEs in the network and all the devices are equipped with the single antenna, the channel is expressed in a 22 matrix $\mathbf{H}$ that 


$$
\mathbf{H}=\left[\begin{array}{ll}
\mathrm{h}_{\mathrm{DL}, 11} & \mathrm{~h}_{\mathrm{DL}, 21} \\
\mathrm{~h}_{\mathrm{DL}, 12} & \mathrm{~h}_{\mathrm{DL}, 22}
\end{array}\right]
$$

According to the (1) the downlink in this transmission for this network can be expressed as

$$
\begin{aligned}
& {\left[\begin{array}{c}
\mathrm{y}_{\mathrm{UE}_{1}} \\
\mathrm{y}_{\mathrm{UE}_{2}}
\end{array}\right]=\left[\begin{array}{cc}
\mathrm{h}_{\mathrm{DL}, 11} & \mathrm{~h}_{\mathrm{DL}, 21} \\
\mathrm{~h}_{\mathrm{DL}, 12} & \mathrm{~h}_{\mathrm{DL}, 22}
\end{array}\right]\left[\begin{array}{cc}
\mathrm{w}_{11} & \mathrm{w}_{21} \\
\mathrm{w}_{12} & \mathrm{w}_{22}
\end{array}\right]\left[\begin{array}{c}
\mathrm{d}_{1} \\
\mathrm{~d}_{2}
\end{array}\right]+\left[\begin{array}{c}
\mathrm{n}_{\mathrm{UE}_{1}} \\
\mathrm{n}_{\mathrm{UE}_{2}}
\end{array}\right]} \\
& \left\{\begin{array}{c}
\mathrm{y}_{\mathrm{UE}_{1}}=\left(\mathrm{h}_{\mathrm{DL}, 11} \mathrm{w}_{11}+\mathrm{h}_{\mathrm{DL}, 21} \mathrm{w}_{12}\right) \mathrm{d}_{1}+\left(\mathrm{h}_{\mathrm{DL}, 11} \mathrm{w}_{21}+\mathrm{h}_{\mathrm{DL}, 21} \mathrm{w}_{22}\right) \mathrm{d}_{2}+\mathrm{n}_{\mathrm{UE}_{1}} \\
\mathrm{y}_{\mathrm{UE}_{2}}=\left(\mathrm{h}_{\mathrm{DL}, 12} \mathrm{w}_{11}+\mathrm{h}_{\mathrm{DL}, 22} \mathrm{w}_{12}\right) \mathrm{d}_{1}+\left(\mathrm{h}_{\mathrm{DL}, 12} \mathrm{w}_{21}+\mathrm{h}_{\mathrm{DL}, 22} \mathrm{w}_{22}\right) \mathrm{d}_{2}+\mathrm{n}_{\mathrm{UE}_{2}}
\end{array}\right.
\end{aligned}
$$

where the useful signals for $\mathrm{UE}_{1}$ are expressed by $\left(\mathrm{h}_{\mathrm{DL}, 11} \mathrm{~W}_{11}+\mathrm{h}_{\mathrm{DL}, 21} \mathrm{~W}_{12}\right) \mathrm{d}_{1}$ and interferences are $\left(\mathrm{h}_{\mathrm{DL}, 11} \mathrm{w}_{21}+\mathrm{h}_{\mathrm{DL}, 21} \mathrm{w}_{22}\right) \mathrm{d}_{2}$. For $\mathrm{UE}_{2}$, the useful signals are $\left(\mathrm{h}_{\mathrm{DL}, 12} \mathrm{w}_{21}+\mathrm{h}_{\mathrm{DL}, 22} \mathrm{w}_{22}\right) \mathrm{d}_{2}$ and interferences are $\left(\mathrm{h}_{\mathrm{DL}, 12} \mathrm{w}_{11}+\mathrm{h}_{\mathrm{DL}, 22} \mathrm{w}_{12}\right) \mathrm{d}_{1}$. Therefore, the maximum allowed power of the eNBs is $\mathrm{P}_{\mathrm{eNB}}$ and here we restrict power allocations for each eNB as

$$
\left\{\begin{array}{l}
\left\{\left(\mathrm{w}_{11} \mathrm{~d}_{1}+\mathrm{w}_{11} \mathrm{~d}_{2}\right)^{2}\right\} \leq \mathrm{P}_{\mathrm{eNB}_{1}} \\
\left\{\left(\mathrm{w}_{12} \mathrm{~d}_{1}+\mathrm{w}_{22} \mathrm{~d}_{2}\right)^{2}\right\} \leq \mathrm{P}_{\mathrm{eNB}_{2}}
\end{array}\right.
$$

Where $\mathrm{P}_{\mathrm{eNB}}=\mathrm{P}_{\mathrm{eNB}_{1}}+\mathrm{P}_{\mathrm{eNB}_{2}}$

According to the zero-forcing pre-coding algorithm, only under the circumstance that all the interference is removed the multi-user transmissions can be reliable, which means the following equalities must be satisfied

$$
\left\{\begin{array}{l}
\mathrm{h}_{\mathrm{DL}, 11} \mathrm{~W}_{21}+\mathrm{h}_{\mathrm{DL}, 21} \mathrm{~W}_{22}=0 \\
\mathrm{~h}_{\mathrm{DL}, 12} \mathrm{~W}_{11}+\mathrm{h}_{\mathrm{DL}, 22} \mathrm{~W}_{12}=0^{(12)}
\end{array}\right.
$$

(11) and (12) is sufficient to define the weights in the pre-coding matrix $\mathbf{W}$ and it is possible to find an optimization value of this matrix to maximize the sum rates. According to the relationship between the information rate and SNR for individual UE, the rate should satisfy 


$$
\left\{\begin{array}{l}
\mathrm{R}_{1} \leq \log _{2}\left(1+\frac{\left|\mathrm{h}_{\mathrm{DL}, 11} \mathrm{~W}_{11}+\mathrm{h}_{\mathrm{DL}, 21} \mathrm{~W}_{12}\right|^{2}}{2}\right) \\
\mathrm{R}_{2} \leq \log _{2}\left(1+\frac{\left|\mathrm{h}_{\mathrm{DL}, 12} \mathrm{~W}_{21}+\mathrm{h}_{\mathrm{DL}, 22} \mathrm{~W}_{22}\right|^{2}}{2}\right)^{(13)}
\end{array}\right.
$$

where $R_{1}$ and $R_{2}$ denote the rate for $\mathrm{UE}_{1}$ and $\mathrm{UE}_{2}$ respectively. And the sum-rate capacity for the base station coordination is then

$$
\mathrm{C}_{\mathrm{BSC}}^{\mathrm{DL}}=\log _{2}\left(1+\frac{\left|\mathrm{h}_{\mathrm{DL}, 11} \mathrm{w}_{11}+\mathrm{h}_{\mathrm{DL}, 21} \mathrm{w}_{12}\right|^{2}}{2}\right)+\log _{2}\left(1+\frac{\left|\mathrm{h}_{\mathrm{DL}, 12} \mathrm{w}_{21}+\mathrm{h}_{\mathrm{DL}, 22} \mathrm{w}_{22}\right|^{2}}{2}\right)
$$

Where the subscript BSC denotes the base station coordination. The uplink transmission can be modeled as

$$
\underbrace{\left[\begin{array}{c}
\mathrm{y}_{\mathrm{eNB}_{1}} \\
\mathrm{y}_{\mathrm{eNB}_{2}}
\end{array}\right]}_{\mathbf{y}_{\mathrm{eNB}}}=\underbrace{\left[\begin{array}{ll}
\mathrm{h}_{\mathrm{DL}, 11} & \mathrm{~h}_{\mathrm{DL}, 21} \\
\mathrm{~h}_{\mathrm{DL}, 12} & \mathrm{~h}_{\mathrm{DL}, 22}
\end{array}\right]}_{\mathbf{H}} \underbrace{\left[\begin{array}{c}
\mathrm{x}_{\mathrm{UE}_{1}} \\
\mathrm{x}_{\mathrm{UE}_{2}}
\end{array}\right]}_{\mathbf{x}_{\mathrm{UE}}}+\underbrace{\left[\begin{array}{c}
\mathrm{n}_{\mathrm{eNB}_{1}} \\
\mathrm{n}_{\mathrm{eNB}_{2}}
\end{array}\right]}_{\mathbf{n}_{\mathrm{eNB}}}
$$

where $\mathbf{H}=\left(\mathbf{h}_{1} \mathbf{h}_{2}\right)$ is the conjugate transpose of the downlink MIMO BC channel $\mathbf{H}$. Here we restrict the individual power $\left\{\left(\mathbf{x}_{\mathrm{UE}, \mathrm{i}}\right)^{2}\right\}=\mathrm{P}_{\mathrm{UE}, \mathrm{i}}$ for the ith UE, and $\mathrm{P}_{\mathrm{UE}}=\mathrm{P}_{\mathrm{UE}, 1}+\mathrm{P}_{\mathrm{UE}, 2}$, where $\mathrm{P}_{\mathrm{UE}}$ is the maximum allowed power of UEs. Then the capacity region of the MIMO MAC uplink transmission is the union of

$$
\mathrm{C}_{\mathrm{MAC}}\left(\mathrm{P}_{\mathrm{UE}} ; \mathbf{H}^{*}\right) \cup\left\{\begin{array}{l}
\mathrm{R}_{1} \leq \log _{2} \operatorname{det}\left(1+\mathbf{h}_{1}^{*} \frac{\mathrm{P}_{\mathrm{UE}, 1}}{\sigma^{2}} \mathbf{h}_{1}\right) \\
\mathrm{R}_{2} \leq \log _{2} \operatorname{det}\left(1+\mathbf{h}_{2}^{*} \frac{\mathrm{P}_{\mathrm{UE}, 2}}{\sigma^{2}} \mathbf{h}_{2}\right) \\
\mathrm{R}_{1}+\mathrm{R}_{2} \leq \log _{2} \operatorname{det}\left(\mathbf{I}+\mathbf{h}_{1}^{*} \frac{\mathrm{P}_{\mathrm{UE}, 1}}{\sigma^{2}} \mathbf{h}_{1}+\mathbf{I}+\mathbf{h}_{2}^{*} \frac{\mathrm{P}_{\mathrm{UE}, 2}}{\sigma^{2}} \mathbf{h}_{2}\right)
\end{array}\right.
$$

where $R_{1}, R_{2}$ are the rates for two UEs separately, $\mathbf{h}_{1}$ and $\mathbf{h}_{2}$ are the first and the second column of $\mathbf{H}$. We can also express the sum-rate capacity in the uplink phase as 


$$
\mathrm{C}_{\mathrm{BSC}}^{\mathrm{UL}}=\log _{2} \operatorname{det}\left(\mathbf{I}_{2}+\mathbf{h}_{1} \frac{\mathrm{P}_{\mathrm{UE}, 1}}{2} \mathbf{h}_{1}+\mathbf{h}_{2} \frac{\mathrm{P}_{\mathrm{UE}, 2}}{2} \mathbf{h}_{2}\right)
$$

\section{Numerical Results}

We run numerical simulation to compare the achievable capacities of base station coordination with the conventional system. The channel gains are all assumed ibid. complex Gaussian $\sim C N(0,1)$, which indicates the ideal Rayleigh fading environment.

The noise in the network are assumed to be additive and with variance ${ }^{2}=0 \mathrm{dBW}$. For static channel coefficients, the expected capacity is the average of sum-rate capacities in each channel realization (1000 times). For simplicity, the power levels are assumed to the same for all the eNBs or UEs. i.e. $\mathrm{P}_{\mathrm{eNB}, 1}=\mathrm{P}_{\mathrm{eNB}, 2}=\frac{1}{2} \mathrm{P}_{\mathrm{eNB}}$ and $\mathrm{P}_{\mathrm{UE}, 1}=\mathrm{P}_{\mathrm{UE}, 2}=\frac{1}{2} \mathrm{P}_{\mathrm{UE}}$

The independent variable chosen here is $\mathrm{P}_{\mathrm{eNB}}$ (or $\mathrm{P}_{\mathrm{UE}}$ in the uplink phase), since the noise is assumed to be unit, $\mathrm{P}_{\mathrm{eNB}}\left(\mathrm{P}_{\mathrm{UE}}\right)$ is also an index of SNR on each parallel channel.

From Figure 3 and 4 the advantage of base station coordination over the conventional time divided transmission is clearly shown. In the downlink phase, in the case of a high SNR for every $3 \mathrm{~dB}$ increase in SNR, the base station coordination with zero-forcing precoding can reach two times of the sum-rate capacity gain $(2 \mathrm{bps} / \mathrm{Hz})$ compared to the conventional system (1 bps/HZ); in the case of a low SNR, the capacity of base station coordination is nearly the same or even lower than the capacity of the conventional system, which indicates that zero-forcing pre-coding, is sub-optimal. The reason is that in base station coordination, the allocated power is not fully used to transmit the useful signal. For example, with $\mathrm{P}_{\mathrm{eNB}}=0 \mathrm{dBW}=1 \mathrm{~W}$, the individual power is $\mathrm{P}_{\mathrm{eNB}, 1}=\mathrm{P}_{\mathrm{eNB}, 2}=\frac{1}{2} \mathrm{P}_{\mathrm{eNB}}=0.5 \mathrm{~W}$. For a

random realization, the channel matrix is

$$
\mathbf{H}=\left(\begin{array}{ccc}
0.1044+0.0093 \mathrm{i} & 0.5572 & 0.4195 \mathrm{i} \\
0.2887+0.39722 \mathrm{i} & 0.1993+0.3443 \mathrm{i}
\end{array}\right)
$$

and the corresponding pre-coding matrix is

$$
\mathbf{W}=\left(\begin{array}{cccc}
0.1717 & 0.2229 \mathrm{i} & 0.1247 & 0.4772 \mathrm{i} \\
0.5799+0.3977 \mathrm{i} & 0.0220 & 0.0708 \mathrm{i}
\end{array}\right)
$$

We can calculate that the actual transmit power of the $1^{\text {st }} \mathrm{eNB}$ is $0.3224 \mathrm{~W}$, and the actual transmit power of $2^{\text {nd }} \mathrm{eNB}$ is $0.5 \mathrm{~W}$. There is a loss of power of $0.1776 \mathrm{~W}$ of base station coordination for this channel realization, which results in a degradation of the sum-rate capacity.

In the base uplink phase, the base station coordination reaches higher capacity both in low and high SNR in the network, the reason for this is the receivers (eNBs) can cooperatively decode so that all the receive signals are considered as useful ones. The system gains from the multiplexing, and the complexity relies on the pre-process or postprocess, which require channel status information on the eNBs.

Figure 5 and 6 show the outage probabilities in the downlink and uplink phase respectively. In the uplink phase it is clear that the coordinated eNBs can reach a lower outage probability than the conventional system. However, in the downlink phase, the 
zero-forcing method does not have gains for base station coordination technology, because of the uplink-downlink duality. We can interpret theoretical sum-rate capacity for the downlink transmission, which is in the same form as equation (17) by replacing power of UEs $\mathrm{P}_{\mathrm{UE}}$ and power of $\mathrm{P}_{\mathrm{eNB}}$.

Finally, Figure 7 and 8 show respectively that ZF pre-coding base station coordination cannot reach the dual theoretical sum-rate capacity bound. To achieve the same sum-rate capacity indicated in the theoretical bound, the base station coordination needs approximately $4 \mathrm{~dB}$ increase in the transmit power, which means ZF is a sub-optimal precoding method. In the other hand, ZF pre-coding base station coordination does not have an outage performance as good as expected in theory because it only cancels the inter-cell interference preliminary but does not make use of it for capacity enhancement.

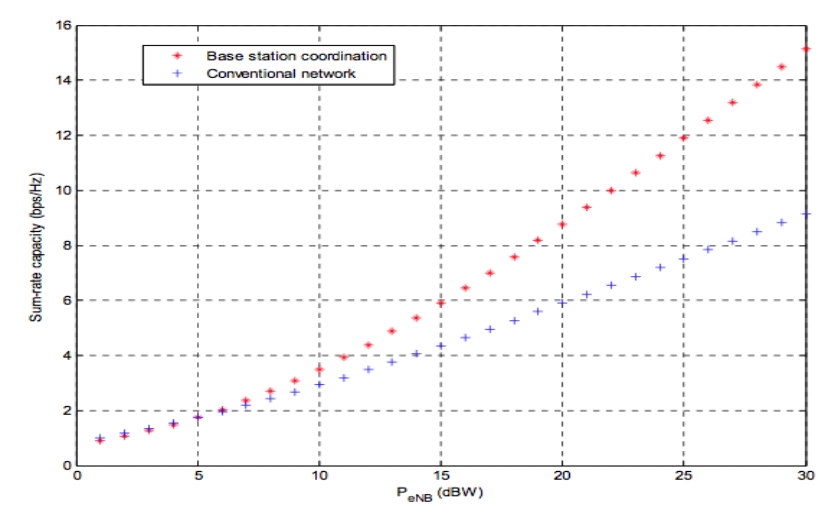

Figure 3. Downlink Sum-Rate Capacity vs. $P_{\mathrm{eNB}}$ Conventional and Base Station Coordination

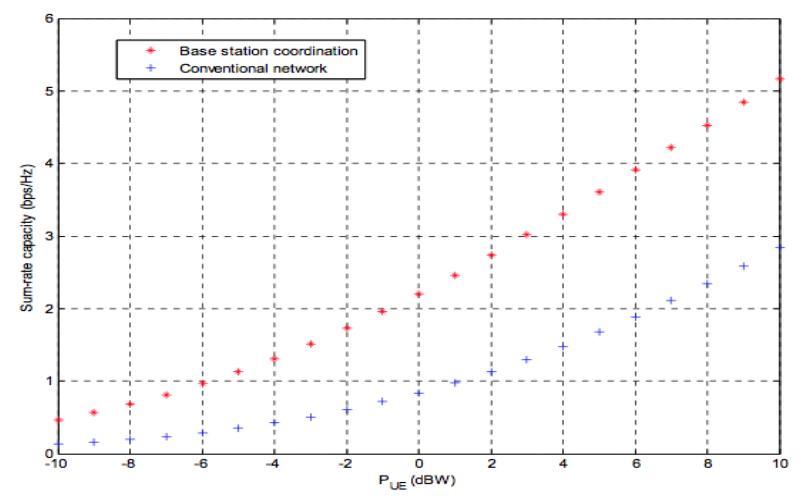

Figure 4. Uplink Sum-Rate Capacity vs. PUE Conventional and Base Station Coordination 


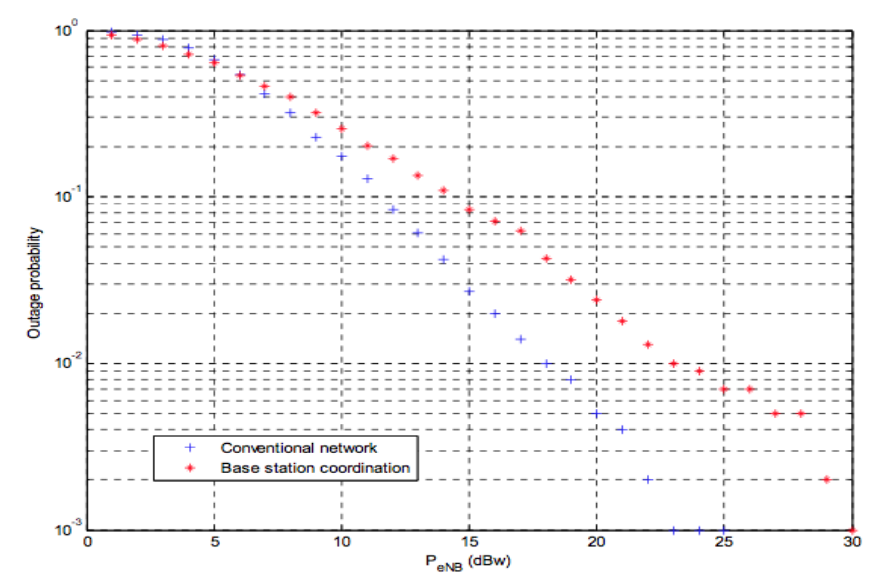

Figure 5. Downlink Outage Probability (at $R=2 b p s / H z)$ vs. PeNB Conventional and Base Station Coordination

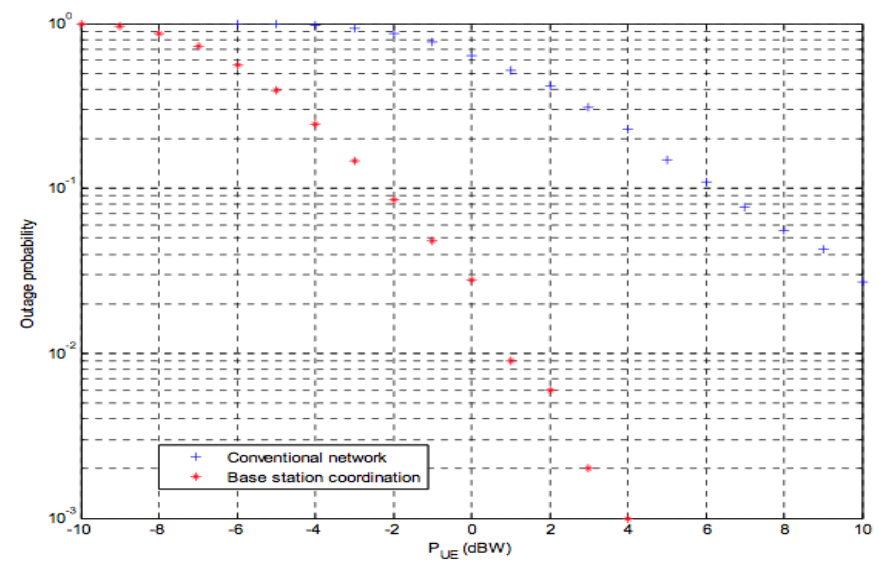

Figure 6.Uplink Outage Probability (at $\mathrm{R}=1 \mathrm{bps} / \mathrm{Hz}$ ) vs. PUE Conventional and Base Station Coordination

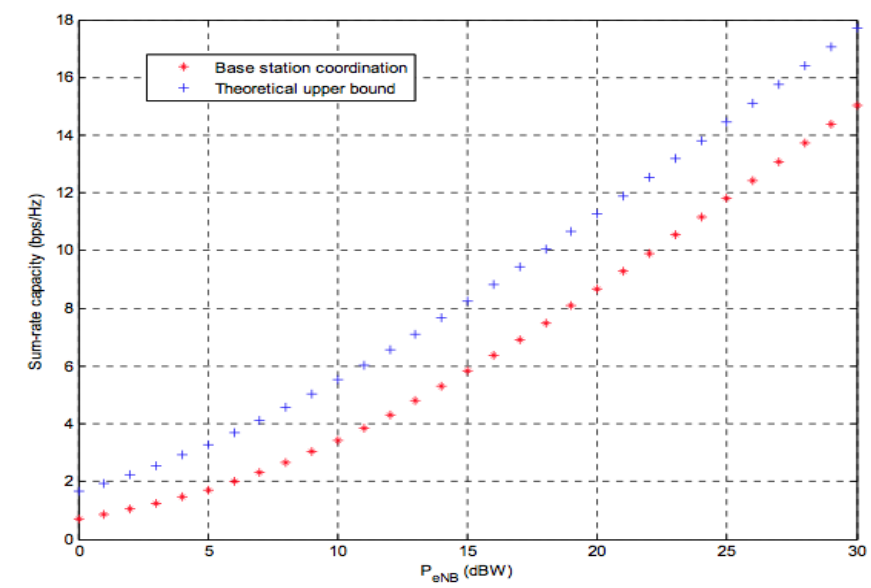

Figure 7. Downlink Sum-Rate Capacity vs. PeNB Theoretical and ZeroForcing Base Station Coordination 


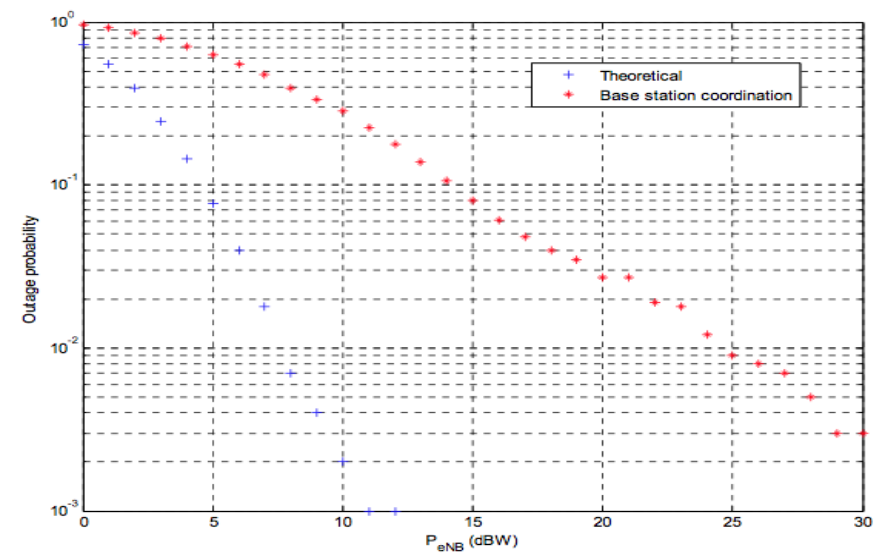

Figure 8. Downlink Outage Probability (at $\mathrm{R}=2 \mathrm{bps} / \mathrm{Hz}$ ) vs. PeNB Theoretical and Zero-Forcing Base Station Coordination

\section{Conclusions}

This paper evaluates the base station coordination technology. With multi-cell processing, the adjacent base station can form a virtual antenna array and then be modeled as MIMO MAC and MIMO BC both in uplink and downlink phases respectively. We can calculate the theoretical bound of multi-user MIMO sum-rate capacity. Zero forcing precoding is chosen to realize multi-user transmission in the downlink phase in the base station coordination scheme. In an application of these theories in a two-cell system, intercell interference is perfectly eliminated and the capacity enhancement comparing to the conventional system is proven in the case of a relatively high SNR.

\section{References}

[1] 3GPP TS21.101 "Overview of 3GPP Release 10 Summary of all Release 10 Features" (2011) April, http://www.3gpp.org/ftp/Information/WORK_PLAN/Description_Releases/

[2] X. Zhang, "Inter-cell Interference Coordination Based on Softer Frequency Reuse in OFDMA Cellular networks", International Conference on Neural Networks and Signal Processing, Shanghai, China, (2008) June, pp. 7-11.

[3] S. Shim, J. Kwak, R. W. Heath Jr., and J. Andrews, "Block diagonalization for multi-user MIMO with other cell interference,” IEEE Trans. Wireless Commun., vol. 7, no. 7, (2008) July, pp. 2671-2681.

[4] Q. H. Spencer, A. L. Swindlehurst, and M. Haardt, "Zero-forcing methods for downlink spatial multiplexing in multiuser MIMO channels," IEEE Trans. Signal Process., vol. 52, no. 2, (2004) February, pp. 461-471.

[5] P. Komulainen, "Downlink assisted uplink zero forcing TDD multiuser MIMO systems", in IEEE WCNC, (2009), pp. 1-6.

[6] C. Suh, M. Ho, and D. N. C. Tse, "Downlink interference alignment," IEEE Trans. Commun., vol. 59, no. 9, (2011), September, pp. 2616-2626.

[7] A. Goldsmith, Editor, Cambridge University Press , "Wireless Communications", (2005).

[8] C. B. Peel, Q. H. Spencer, A. L. Swindlehurst and M. Haardt, "An introduction to the multi-user MIMO downlink", IEEE Commun. (2004), pp. 60-67.

[9] H. Holma and A. Toskala, Editor, John Wiley \& Sons, Inc., "WCDMA for UMTS: Radio Access for Third Generation Mobile Communications", New York, NY, USA, 1st edition, (2001). 


\section{Authors}

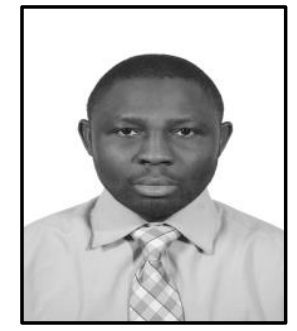

Bertrand Deflandre Moubagou PhD., research Scholar at Beijing University of Posts and Telecommunications, China. In July 2008 and June 2011, He received respectively his Bachelor Engineering in Communication Engineering and Master Degree in Information and Communication Systems from the same university. His research interests include: Wireless Communication, Cooperative Communication, and Radio Resource Management in LTEAdvanced.

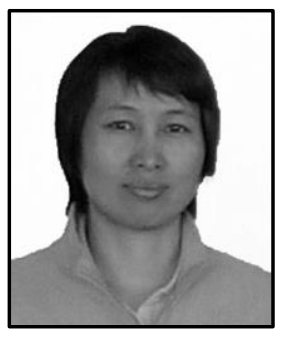

Yongyu Chang, received her $\mathrm{PhD}$ from Beijing University of Posts and Telecommunications, Beijing, China, in July 2005. Since then, she has worked for the Wireless Theory and Technology Laboratory of School of Information and Communication Engineering. She has been engaged in the research of theories and key technologies in the mobile communication area for many years. As a director or key member, she has made remarkable achievements by leading or participating in many research programs, some of which are supported by the government, such as the China National 973 Program and the China National 863 Program. Other programs are company-sponsored projects. Her current research is focused on the key technologies and system performance evaluation for $4 \mathrm{G} / 5 \mathrm{G}$ wireless networks. 
International Journal of Future Generation Communication and Networking Vol. 8, No. 2 (2015) 